\title{
Applied Research on AC/DC Integrated Power Supply of Substation
}

\author{
Lu Zhigang ${ }^{1, a}$, Lei Bo ${ }^{1, b}$, Zheng Yongqiang ${ }^{2, c}$
}

${ }^{1}$ State Key Laboratory of HVDC, Electric Power Research Institute, China Southern Power Grid,
Guangzhou, 510080, China

${ }^{2}$ Zhuhai Watt Electric Equipment Co., LTD, Zhuhai, 519080,China

aemail: luzg@csg.cn, bemail:leibo@csg.cn, cemail:watt_KY@163.com

Keywords: AC/DC integrated power supply; battery; condition monitoring; IEC 61850

\begin{abstract}
According to the problem that the intelligence is not high of the soccer robot, using the mechanical theory as a guide, making some mechanical analyses and calculations on the pressure and transmutation states of chip kick mechanics, and conducting optimal design too, then making the structure of chip kick mechanics more and more rationalization. Experiments show that the new soccer robot controller features a quick response and high servo rigidity, and provide a kind of method for improving and perfecting the soccer robot control system, at the same time, filling the needs of producing.
\end{abstract}

\section{Introduction}

The AC/DC integrated power supply of substation consists of the substation AC power supply, DC operational power supply, UPS and communication power supply, etc. Based on DC power system, all its sub-power systems are under unified design, planning, O\&M and management to provide stable and reliable power for relay protection, server, communication, emergency lighting, etc. It is an indispensable part for a substation [1]-[2].

\section{System configuration}

Centered on DC operational power supply, the AC/DC integrated power supply of substation integrates the substation AC power supply (AC), DC operational power supply (DC), AC uninterruptable power supply for power (UPS), DC conversion power supply for communication (DC - DC), shares the battery of DC operational power supply and conducts centralized monitoring, as shown in the schematic figure 1.

In the system, the substation AC power is mainly composed of AC incomer, AC feeder, power measurement and AC monitoring. It is equipped with the ATS for automatic transfer between the two AC incomer power supplies to ensure AC output distribution and long-term stable operation of AC/DC integrated power supply. The DC operational power, as the core of AC/DC integrated power supply, is mainly composed of the rectifier unit, battery unit and DC feeder unit. The UPS mainly serves to supply power to key loads with high requirements on power supply in the substation, such as computer monitoring, printer, etc. The INV power source mainly serves to supply power to loads with general requirements on power supply in the substation, for example, emergency lighting. The DC/DC communication power supply employs the DC220/110V DC bus power supply as the input power supply of communication rectifier module, and shares the batteries with DC power supply to provide reliable working DC power to exchanger and other communication equipment in the substation. The monitoring system stores all data of the substation power supply and is connected to the background through a RJ45 port with IEC-61850 protocol to realize the four-remote control function of the AC/DC integrated power supply of substation [3]. 


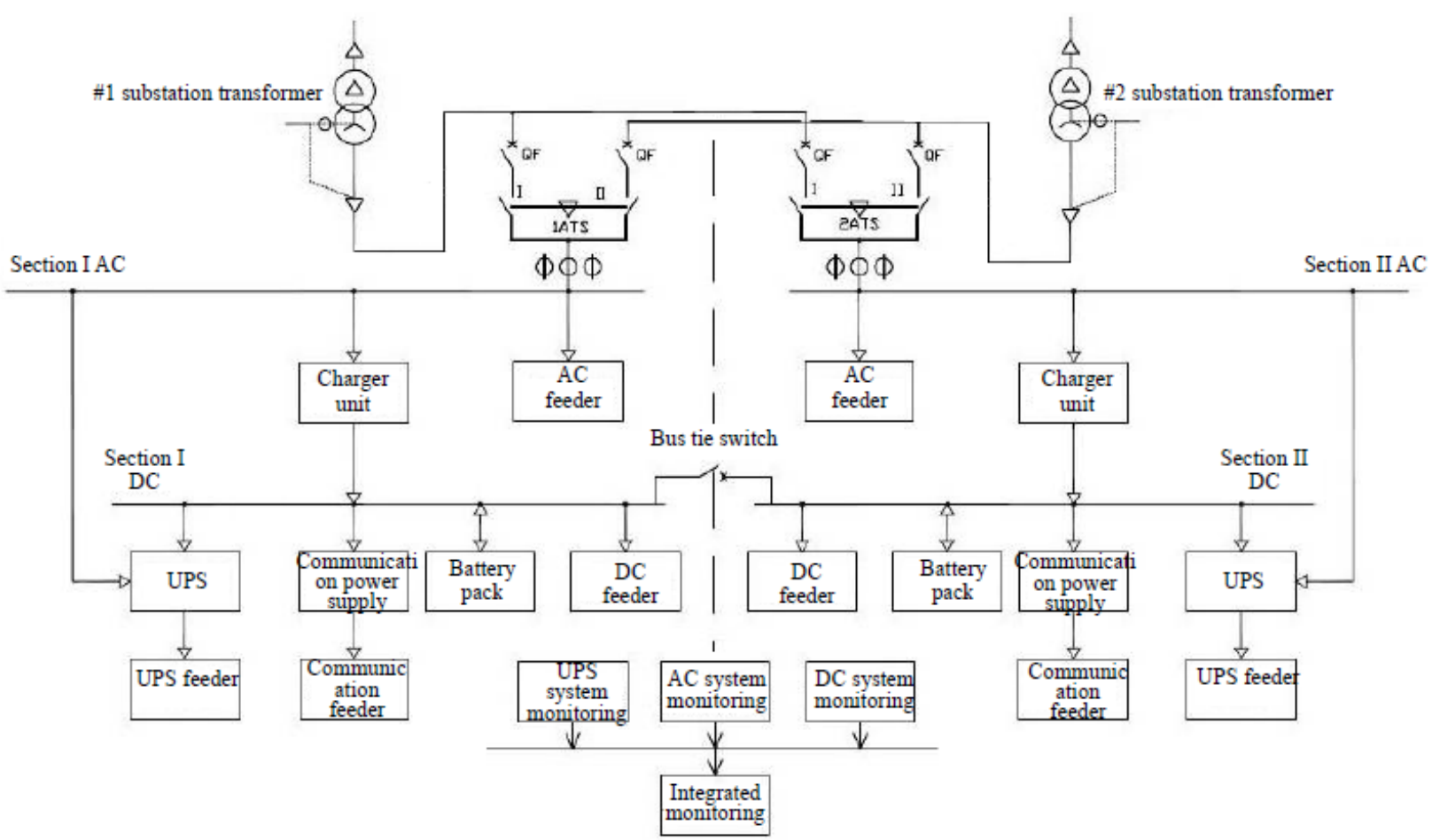

Fig.1. Schematic diagram of AC/DC integrated power supply of substation

\section{Design principles}

a) Reliability. The integrated monitoring system and the measurement and control unit of each sub-system are independent and run in parallel. The failure or malfunction of integrated monitoring system does not affect the normal operation of subsystems, which is beneficial to the power supply reliability of the whole power system.

b) Intelligence. It provides decentralized control and centralized management on each sub-power supply system through field bus and realizes digital communication with the automation system through the Ethernet interface and the IEC61850 protocol. Besides, it owns the capacity of intelligent control analysis, which could provide data for the condition-based maintenance.

c) Uniformity. The AC/DC integrated power supply of substation is under unified design, production, commissioning, operation and maintenance and employs uniform external interface and panel appearance.

\section{Main features}

a) Resource economy and optimized configuration. With integrated DC operational power battery, UPS battery, and communication power battery, unified design and reasonable panel arrangement, it reduces repeated configuration, saves the space, and makes positive contribution to the improvement of environmental quality.

b) Uniform language and open system. All data and information of subsystem employ the uniform information model and programming language. Ethernet interface and IEC61850 protocol are employed for communication with upper computer system. It realizes interchangeability and interoperability among intelligent equipment of different manufacturers. The substation power system becomes an open system with uniform language.

c) High integration and high intelligence. Different parts of the system are interconnected with network and connected to the integrated comprehensive monitoring device, which conducts real-time monitoring on the operation conditions. Based on comparison and analysis of real-time and historical data, it makes scientific evaluation and analysis on the operation conditions and performance to provide detailed data support for the operation and maintenance and further provide necessary technical preparation and practice for scientific management of AC/DC integrated power 
supply of substation and construction of digital substations.

d) Unified management, efficiency and reliability. The substation AC/DC integrated power supply conducts unified management on all subsystems, which optimizes the operation process and human resource allocation, reduces the repeated equipment configuration, and lowers equipment investment and O\&M cost. Also, by employing unified design, distributed implementation and graphic interface display, and allowing different systems viewing operation information on the same interface, it can conduct intelligent control on all sub-power systems and thus greatly improve the operation reliability.

e) Expert system and IEC61850 communication. The substation AC/DC integrated power supply monitoring may also have embedded expert system and IEC61850 communication model building function, as shown in figure 2, to conduct real-time record and analysis on the system operation data, and make forecast and judgment on the trend of system operation, which greatly reduces the system operation complexity and maintenance difficulty[4].

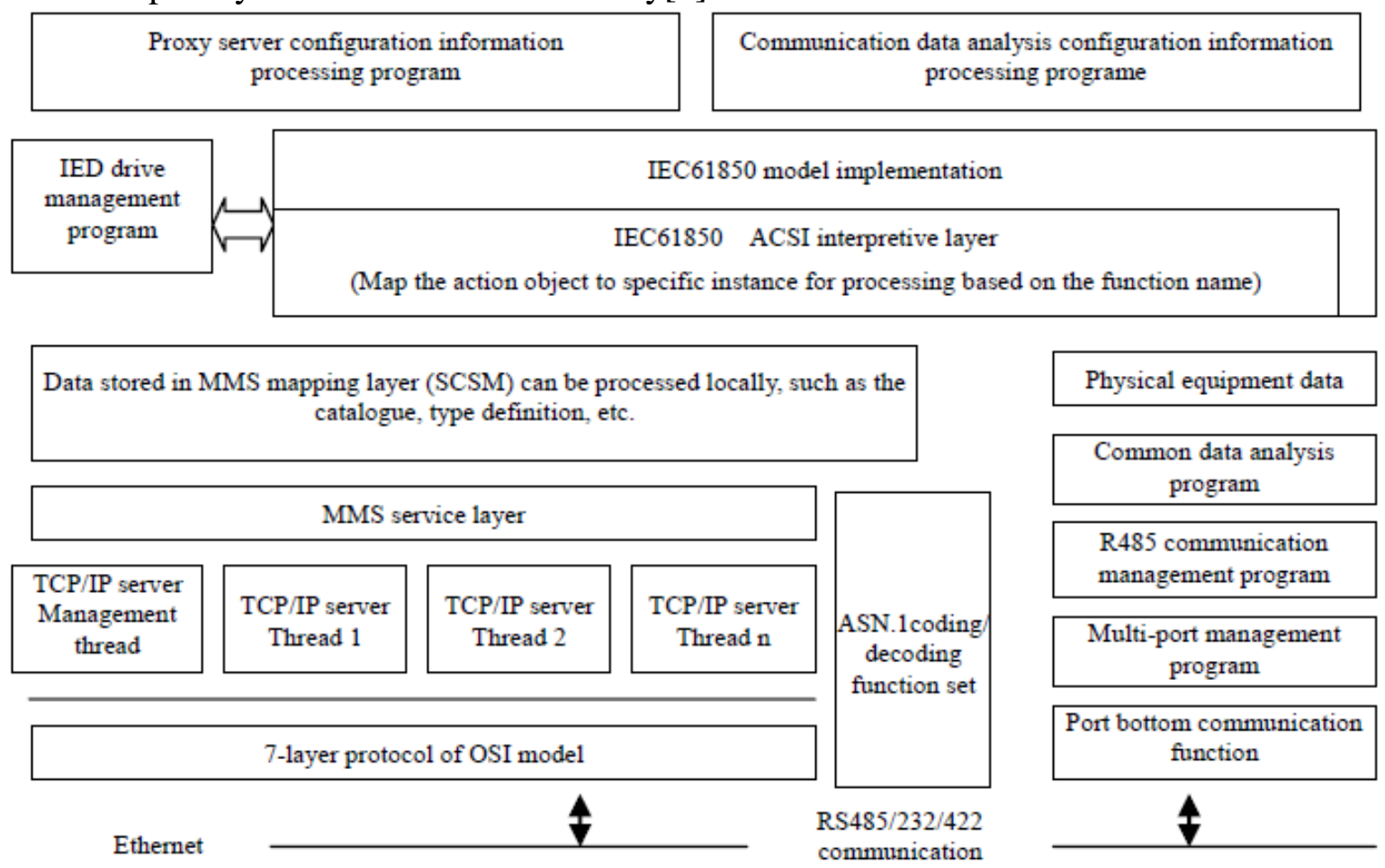

Fig.2. IEC 61850 communication model building

\section{Status monitoring}

a) Present application. The substation AC/DC integrated power supply includes the AC power supply, DC operational power supply, AC uninterruptible power supply, inverter power supply and communication power supply. As a large system with various kinds of devices, it has many potential points of failure. Besides, as there is a variety of auxiliary equipment inside each power supply, it is difficult to conduct unified installation, commissioning and maintenance among different power supplies. Therefore, its O\&M is facing both great workload and difficulty.

Most of existing conventional monitoring systems only monitor part of electrical parameters of power supply, such as various input voltage, input current, bus voltage, battery voltage and current, feeder switch condition, DC insulation, etc., with comparatively less focus on monitoring of the overall power system operation, most of which are even merely post-incident reporting. It makes O\&M only passive and late remedy rather than active prevention.

b) Development direction. The condition monitoring of substation AC/DC integrated power supply is mainly to collect and analyze various dataset and conduct control and management on the overall system based on system operation conditions, as shown in figure 3 . In addition, it serves to upload the power supply system information to background with the communication protocol 
developed by customers to adapt to various operation modes of the power supply system. The monitoring unit usually has different modes of display, such as the button type, touch screen type, etc. and the user can make free selection according to their needs.

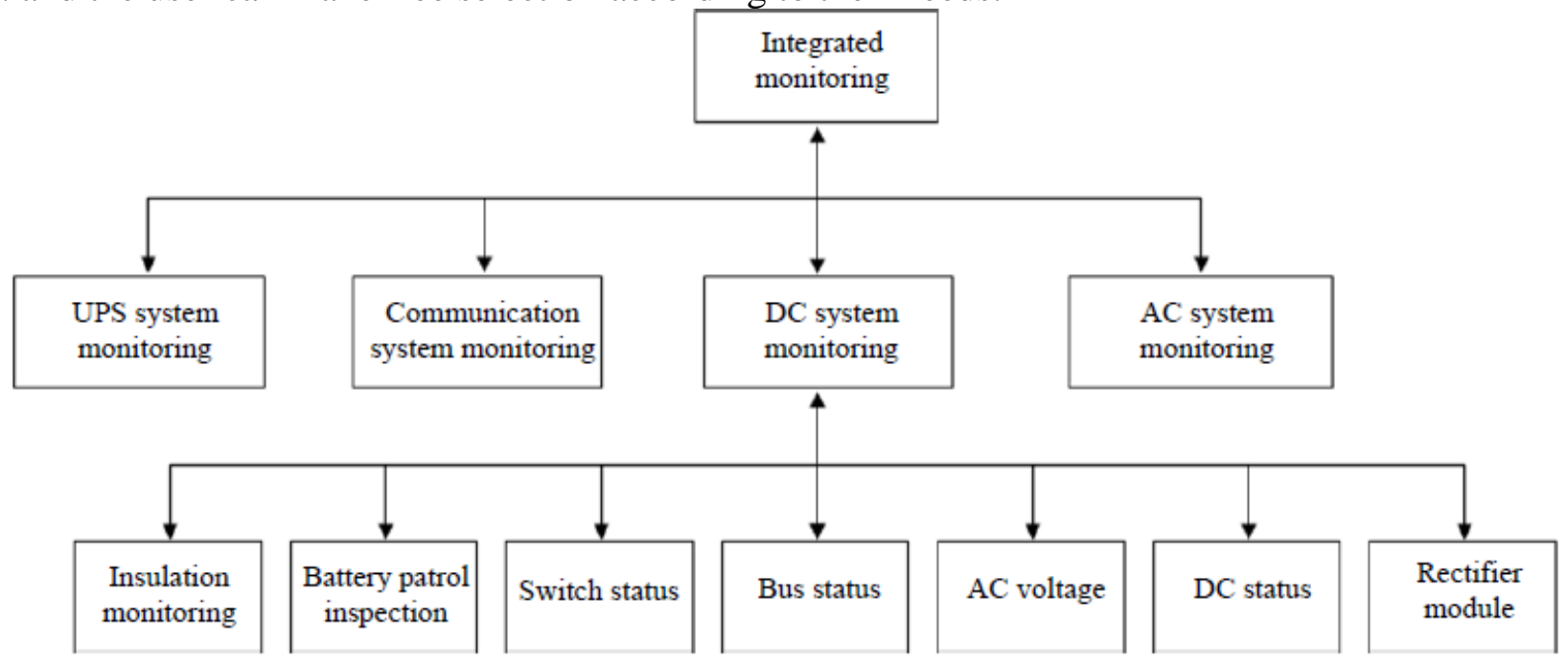

Figure 3: Principles of monitoring system

The existing monitoring unit detects and reports the operation data of power supply and part of equipment. Because there are various alarm thresholds, if there is a failure, most of analog variables will generate alarms. Besides, various state variables will also generate alarms in cases of change. The method can only be used to find the fault, but is far from analysis.

Considering the needs of long-term development of substation power system, the expert system should be employed, which takes periodic snapshots and then compares and analyzes the operation data and tend of the power supply and various other equipment based on continuous snapshots showing recent operation of the system, so as to learn the health status of the whole system and provide reference and basis for operation and maintenance. Besides, the system owns the expansion capability and can have embedded expert algorithm developed by O\&M personnel based on long-term fault judgment and O\&M experience to develop expert diagnostic data and realize continual improvement of system functions.

For example, the comparison and analysis of charger module voltage, closing busbar voltage and battery voltage can identify which voltage has deviation and be further used to analyze whether the charger, battery or collection device is abnormal; the communication failure rate of a specific charger can be used to analyze whether the communication lines or communication module is abnormal; the change in feeder insulation resistance can be used to analyze and forecast the risk of insulation deterioration, etc. The expert system can significantly increase the maintenance efficiency, reduce the system risk, and accumulate knowledge on substation maintenance.

\section{Battery Management}

a) Configuration principle. The valve-regulated lead-acid battery is normally used in the substation power system. Besides, the new lithium battery with a long life can also be used. To effectively meet the needs for redundant power supply control and protection, two sets of high frequency charging devices and two packs of batteries are provided for 220-500kV substation. Also, to meet the needs of unattended DC redundancy power supply, part of the $110 \mathrm{kV}$ substations are also provided with two sets of high frequency charging batteries and two packs of batteries.

Batteries can be installed centrally or separately. In central installation, the batteries are placed in a central cabinet and then installed together with other cabinets, such as charger cabinet, feeder cabinet, etc.; while in separate installation, the batteries are installed on supports in separate battery room. The customer can choose the appropriate way of installation according to site conditions. Generally separate installation is used when the transformer substation is equipped with the relay protection device cabin. 
b) Operation and maintenance. Regular inspection serves to measure and record the shell temperature and terminal voltage of each battery and reports any value exceeding the limit specified in a timely manner. The current overvoltage standard for power grid in China is that the difference between terminal voltage of a specific battery and the average value is $0.05 \mathrm{~V}$ higher or lower than the specific value.

The daily patrol covers the cleanness, leakage and deformation of battery terminals and shell, consistence between meter indication and actual value, operation of auxiliary components, position of automatic circuit breaker, status of fuse, ventilation, lighting and firefighting equipment of battery room, completeness and indication of operation and monitoring signals in each branch line, operation of microcomputer monitoring units, clarity and completeness of device markings, the closing busbar and DC busbar voltage being in specific range or not, etc.

c) Main factors affecting battery life. For batteries under long-term floating charge state, it is impossible to make accurate judgment whether cracking or hydration occurs inside. It is very inappropriate to charge the batteries for a long time without discharging, which will greatly shorten the actual backup power supply time and significantly reduce the service life of battery. The main reason lies in that the passivated battery anode plate will increase the battery internal resistance to some extent and finally reduce the actual battery capacity to a value far below the standard capacity. The main indication of battery hydration is that when the battery discharges, the voltage will quickly decline to the cut-off value. Floating charge voltage is very normal during operation, but the daily charge and discharge strategy is the key factor affecting battery life. The long-term floating charge will reduce active material of battery and reduce the battery capacity. The general solution the problem is to replace the battery in time.

Battery overcharging will generate a certain degree of redox reaction and reduce the effective area of electrode plate. Besides, overcharging can also result in electrolyte overflow and emerging of oxide, which will reduce the insulation performance of battery and cause battery self-discharge. In such cases, the maintenance personnel is required to reduce the charging voltage and current, and check the blocking of battery safety valve and the setting of different accumulator parameters. In cases of over-discharge, that is, the output voltage being reduced to zero, a large quantity of lead sulfate inside the battery will be drawn to the cathode surface. As the lead sulfate is a kind of insulator, the battery internal resistance will increase with the growing quantity of sulfate on the cathode plate, which will reduce the performance of the battery charge and discharge and shorten the battery life. Over-discharge usually occurs when the battery is used for power supply after the charging device fails or AC power is lost. It is also a key factor affecting battery life[5].

\section{Conclusion}

In past over 20 years, the substation power supply witnessed the constant development towards stableness, reliability, consistency, and easy maintenance. It requires optimization in various aspects from needs, design to O\&M to realize stableness, interruption, reliability and easy maintenance. The AC/DC integrated power supply of substation will develop into a highly-integrated, highly-intelligent and highly-reliable system with low failure rate requiring little maintenance.

\section{Acknowledgement}

In this paper, the research was sponsored by the State Key Laboratory of HVDC and Scientific Research Project of Technology Research Center of China Southern Power Grid Co. Ltd(Project No. CSGTRC-142021).

\section{References}

[1] Wang Heng. Analysis of Substation Integrated Power Supply Construction [J]. Power Supply Technologies and Applications, 2014(3), 07-07.

[2] Wu Fengting. Solution of Substation AC/DC Integrated Power Supply[J]. Southern Power 
System Technology, 2011, 5 (3), 87-89.

[3] Kong Rongrong and Zhang Lina. A Brief Analysis of Applications and Development of Substation AC/DC Integrated Power Supply [J]. Science-Technology Enterprise, 2015 (5), 190-190.

[4] Chang Qing and Ding Liang. Research on IED Information Modeling Method of Substation Integrated Power Supply Based on IEC61850 [J]. Electron Devices, 2014, 37 (1), 138-142.

[5] Xie Congqian. Study on Operation and Maintenance of Substation DC Power Supply [J]. Mechanical \& Electrical Engineering Technology, 2011, 40 (4), 27-29. 